\title{
Zur Kinetik der Enzyme.
}

Von

S. G. Hedin.

(Der Redaktion zugegangen am 16. September 1908.)

Die Methoden zur Bestimmung der Wirkung der Enzyme sind in vielen Fällen noch sehr mangelhaft. Wo es sich um einfache Reaktionen handelt, z. B. bei der Zersetzung von Estern, oder von Wasserstoffsuperoxyd, läßt sich die Wirkung verhältnismäßig leicht verfolgen, aber in Fällen, wo die Prozesse stufenweise verlaufen oder vielleicht mehrere Prozesse parallel stattfinden, was z. B. wahrscheinlich bei der Zerlegung des Eiweißes zutrifft, da könnte es von vornherein aussichtslos scheinen, den Fortschritt der Reaktion genau verfolgen zu können. Was speziell die Zerlegung des Eiweißes betrifft, wird wahrscheinlich die neue Methode von Sörensen viel mehr leisten als die früher angewandten. ${ }^{1)}$ Diese geben alle falsche Resultate. Bestimmt man z. B. die während einer gewissen Zeit aufgelöste Eiweißmenge, so läßt man außer Rechnung die Veränderungen, welche durch das Enzym während derselben Zeit auf bereits aufgelöstes Eiweiß ausgeführt wurden. Wird irgend welche Fällungsreaktion angewandt, um das unverdaute Eiweiß zu bestimmen, z. B. Fällung unter Aufkochen mit Essigsäure, Fällung mit Gerbsäure oder Phosphorwolframsäure, so vernachlässigt man die enzymatische Arbeit, welche auf die nicht fällbaren Produkte verrichtet wurde. Es leuchtet sofort ein, daß man in diesen Fällen zu falschen Resultaten gelangen würde, wollte man die nach verschiedenen Zeiten stattgehabte Enyzmwirkung einfach der aufgelösten und nicht mehr fällbaren Eiweißmenge proportional setzen. Ebenso unrichtig wäre es, wollte

1) Biochem. Zeitschrift, Bd. VII, S. 45 u. 407, 1907. 
man die in einer gewissen Zeit mit verschiedenen Enzymmengen stattgefundenen Veränderungen in angedeuteter Weise messen. Nur in Ausnahmefällen z. B. bei Überschuß von Substrat kann dies zulässig sein.

Wie Duclaux hervorgehoben hat, ist es aussichtsvoller, anstatt die in gleichen Zeiten stattgehabten Umsätze zu vergleichen, die Zeiten aufzusuchen, in welchen der gleiche Umsatz erhalten wird. ${ }^{1}$ ) Nun haben verschiedene Forscher für mehrere Enzyme ein sehr einfaches Verhältnis gefunden zwischen der Enzymmenge und der für einen gewissen Umsatz nötigen Zeit der Einwirkung. Wenn man verschiedenen Proben, welche die gleiche Substratmenge enthalten, verschiedene Enzymmengen (p) zugibt, so wird der gleiche Umsatz erhalten, wenn die Zeit der Einwirkung (t) den zugegebenen Enzymmengen umgekehrt proportional variiert wird. Man kann auch sagen, daß dem gleichen Werte für $\mathrm{p} \cdot \mathrm{t}$ die gleiche Wirkung entspricht, oder daß der gleiche Umsatz erhalten wird nach dem Verlauf von den gleichen Enzym-Zeiteinheiten. Diese Regel wurde für folgende Enzyme gültig gefunden: Invertin (0'Sullivan und Tompson unter gewissen Bedingungen), ${ }^{2}$ ) Pepsin (Sjöqvist), ${ }^{3}$ ) Lab (Fuld), ${ }^{4}$ ) peptonspaltende Enzyme (Vernon), ${ }^{5}$ ) Fibrinfermente von Schlangengiften (Martin), ${ }^{6}$ ) Trypsin (Hedin), $\left.{ }^{7}\right)$ Pepsin, Lab, Trypsin, Pyocyaneusprotease (Madsen). ${ }^{8}$ )

Was bedeutet nun diese einfache Regel? Ich werde hier eine Betrachtungsweise anwenden, die schon vorher von Ostwald für katalytische Beschleunigung gebraucht worden ist. ${ }^{9}$ ) $\mathrm{Da}$ wir die Abhängigkeit der Reaktionsgeschwindigkeit von der Substratkonzentration nicht kennen, wollen wir die Reaktions-

1) Traité de microbiologie, S. 380.

2) Journ. Chem. Soc. Trans., Bd. LVII, S. 926, 1890.

${ }^{3}$ ) Skand. Arch. f. Physiol., Bd. V, S. 358, 1895.

4) Beitr. z. chem. Physiol. u. Pathol., Bd. II, S. 169, 1902.

5) Journ. of Physiol., Bd. XXX, S. 334, 1903.

6) Ibid., Bd. XXXII, S. 207, 1905.

7) Ibid., Bd. XXXII, S. 468, 1905; Bd. XXXIV, S. 370, 1906.

8) Arrhenius, Immunochemie, Leipzig 1907, S. $46 \mathrm{ff}$.

9) Zeitschrift f. physik. Chemie, Bd. II, S. 134, 1888. 
geschwindigkeit $\frac{\mathrm{dC}}{\mathrm{dt}}$ als eine unbekannte Funktion von der Konzentration $\mathrm{f}(\mathrm{C})$ schreiben. Wir haben also $-\frac{\mathrm{dC}}{\mathrm{dt}}=\mathrm{k} \cdot \mathrm{f}(\mathrm{C})$, wo $\mathrm{k}$ der von der Enzymmenge abhängige Geschwindigkeitskoeffizient bedeutet.

Durch Integration $z$ wischen den Zeiten $o$ und $t$ und den zugehörigen Konzentrationen $\mathrm{C}$ und $\mathrm{C}_{1}$ bekommen wir $\varphi(\mathrm{C})-\varphi\left(\mathrm{C}_{1}\right)=\mathrm{k} \cdot \mathrm{t}$.

Wenn nun bei einer anderen Enzymmenge der Gang der Zerlegung des Substrates der gleiche wäre, so würde $-\frac{\mathrm{dC}_{\mathrm{C}}}{\mathrm{dt}}=\mathrm{k}^{1} \mathrm{f}(\mathrm{C})$, wo nur der Geschwindigkeitskoeffizient ein anderer ist als vorher. Integrieren wir zwischen $o$ und $t^{1}$ und den Konzentrationen $\mathrm{C}$ und $\mathrm{C}_{1}^{1}$, so bekommen wir $\varphi(\mathrm{C})-\varphi\left(\mathrm{C}_{1}^{1}\right)$ $=\mathrm{k}^{1} \mathrm{t}^{1}$. Wählen wir nun die Zeit $\mathrm{t}^{1}$ so, daß $\mathrm{C}_{1}=\mathrm{C}_{1}^{1}$, d. h., so daß der Umsatz in beiden Fällen der gleiche bleibt, so ist $\mathrm{k} \cdot \mathrm{t}=\mathrm{k}^{1} \cdot \mathrm{t}^{1} \operatorname{oder} \frac{\mathrm{k}}{\mathrm{k}^{1}}=\frac{\mathrm{t}^{1}}{\mathrm{t}}$.

Aus diesen allgemeinen Betrachtungen geht also hervor, daß, wenn mit verschiedenen Enzymmengen die Zerlegung des Substrates dem gleichen Weg folgt, die Zeiten gleichen Umsatzes den Geschwindigkeitskonstanten umgekehrt. proportional sind. Der Beweis, daß der Reaktionsverlauf bei verschiedenen Enzymmengen von Störungen und Nebenreaktionen in der gleichen Weise beeinflußt worden ist, muß für jeden Fall geführt werden. $\mathrm{Da}$ unter der genannten Bedingung die Geschwindigkeitskoeffizienten sich umgekehrt, wie die Zeiten gleichen Umsatzes verhalten, so kann der fragliche Beweis als geliefert angesehen werden, wenn es sich herausstellt, daß die den verschiedenen Enzymmengen $\mathrm{p}$ und $\mathrm{p}^{1}$ entsprechenden Zeiten gleichen Umsatzes $t$ und $t^{1}$ auf verschiedene Stadien der Reaktion zu einander ein konstantes Verhältnis zeigen. Nun hat es sich bei der Einwirkung von Trypsin auf Casein gezeigt, daß $p \cdot t=p^{1} \cdot t^{1}$ oder $\frac{t^{1}}{t}=\frac{p}{p^{1}}=$ konstant. Dies gilt für verschiedene Stadien der Reaktion, $d . h$. bei ver- 
schiedenen Werten für $\mathrm{p} \cdot \mathrm{t}$. Als Beleg mögen aus meiner zitierten Arbeit folgende Ziffern angeführt werden. Die in die verschiedenen Kolumnen unter den Werten von $\mathrm{p} \cdot \mathrm{t}$ eingetragenen

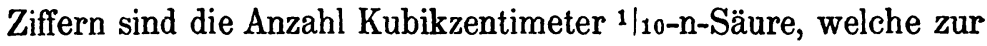
Neutralisierung des aus gleichen Volumina des Gerbsäurefiltrates nach Kjeldahl erhaltenen Ammoniaks erforderlich waren.

\begin{tabular}{r|c|c|c|c|c|c|c|c}
\hline \hline $\mathrm{pt}=$ & 1 & 2 & 2,5 & 5 & 7,5 & 10 & 15 & 20 \\
\hline $\mathrm{t}=1$ & 5,80 & 10,35 & 12,95 & 20,15 & 24,5 & 26,95 & 31,0 & 34,05 \\
2 & 5,85 & 10,75 & 13,25 & 20,40 & 24,65 & 27,8 & 31,7 & - \\
3 & 5,70 & 10,85 & 13,35 & 20,30 & 24,35 & 27,0 & 31,05 & 33,75 \\
4 & 6,10 & 10,75 & 13,15 & 19,90 & 23,80 & 26,95 & 30,75 & 33,55
\end{tabular}

Wir haben also, wenn $\mathrm{p}$ und $\mathrm{p}^{1}$ zwei verschiedene Enzymmengen, $\mathrm{k}$ und $\mathrm{k}^{1}$ die entsprechenden Geschwindigkeitskoeffizienten und $t$ und $t^{1}$ zugehörige Zeiten gleichen Umsatzes bedeuten, nach den obigen Betrachtungen $\frac{k}{k^{1}}=\frac{t^{1}}{t}$. Anderseits geht aus den Versuchen mit Trypsin direkt hervor, daß $\mathrm{p} \cdot \mathrm{t}=\mathrm{p}^{\mathbf{1}} \cdot \mathrm{t}^{\mathbf{1}}$ oder $\frac{p}{p^{1}}=\frac{t^{1}}{t}$. Folglich ist: $\frac{k}{k^{1}}=\frac{p}{p^{1}}$ oder die Geschwindigkeitskoeffizienten sind den Enzymmengen proportional. ${ }^{1}$ )

Für katalytische Reaktionen in einem homogenen Systeme ist die Regel im allgemeinen gültig, daß der Geschwindigkeitskoeffizient der Menge des Katalysators proportional ist, und der Beweis, daß eine Ionengattung bei einer Reaktion als Katalysator wirksam ist, wird in der Weise geführt, daß k der Menge der Ionengattung proportional gefunden wird. Für die durch Enzyme vermittelten Reaktionen ist dieser Beweis bisher nur in Ausnahmefällen geführt worden. So fand Armstrong, daß für kleine Mengen von Maltase (oder Überschuß von Milch-

1) Der Geschwindigkeitskoeffizient gibt den in der Zeiteinheit stattgehabten Umsatz unter der Voraussetzung an, daß die Konzentration des Substrates während der Zeit konstant gehalten wird. 
zucker) der Umsatz der Enzymmenge proportional war, ${ }^{1}$ ) und dasselbe habe ich für die Einwirkung von Trypsin auf einen Überschuß von Casein nachweisen können. ${ }^{2}$ ) Die obige Herleitung des Gesetzes bezieht sich aber nicht nur auf solche Fälle, sondern ist innerhalb des Bereiches gültig, wo der Umsatz für die gleiche Anzahl Enzym-Zeiteinheiten derselbe ist.

Es wird nunmehr von den meisten Forschern angenommen, daß die Enzyme vor ihrer chemischen Wirkung in irgend einer Weise mit dem Substrat sich verbinden. Die mit dem Substrat verbundene Enzymmenge ist also das wirksame Enzym oder die aktive Masse des Enzyms. Wäre nur ein einziges Substrat vorhanden, so würde die Regel, daß die Geschwindigkeitskonstante der zugesetzten Enzymmenge proportional ist, bedeuten, daß die vom Substrate aufgenommene Enzymmenge der zugesetzten proportional ist. Nun sind aber gewisse Enzymreaktionen viel verwickelter. In einigen Fällen sind vom Anfang ab mehrere Substrate zugegen oder es bilden sich während der Wirkung des Enzymes mehrere verschiedene Substrate oder Substanzen, welche das Enzym aufnehmen können. Die verschiedenen Substrate werden mit ungleicher Geschwindigkeit umgesetzt. Die oben gefundene Regel, nach welcher die Reaktionsgeschwindigkeit der Enzymmenge proportional ist, muß also bedeuten, daß bei gleichem Substratgemisch die von jedem Substrat aufgenommene Enzymmenge der zugesetzten Enzymmenge proportional ist, oder daß die Verteilung des Enzyms zwischen den Substraten unabhängig von der zugesetzten Enzymmenge ist.

Nun ist aber das Substratgemisch nach der gleichen Anzahl von $\mathrm{p} \cdot \mathrm{t}$ das gleiche; man kann also sagen, daß die Verteilung des Enzyms bei gleichem Umsatz die gleiche ist unabhängig von der Enzymmenge. Dasselbe gilt bekanntlich für die Verteilung eines löslichen Stoffes zwischen zwei oder mehreren Lösungsmitteln; die verschiedenen Substrate dienen gewissermaßen als Lösungsmittel für das Enzym.

Es leuchtet sofort ein, daß, wenn unter den gebildeten enzymatischen Produkten irgendwelches das Enzym aufnimmt,

i) Proc. Roy. Soc., Bd. LXXIII, S. 500, 1904.

2) Journ. of Physiol., Bd. XXXII, S. 471, 1905. 
aber unter der Einwirkung des Enzyms keine Veränderung leidet, dieses die Enzymwirkung hemmt, aber daß diese Hemmung auf gleiche Umsätze die gleiche bleiben muß. Die Gültigkeit des Gesetzes, daß der Umsatz nach Verlauf der gleichen Anzahl von $p \cdot t$ derselbe ist, wird durch eine solche Hemmung nicht berührt.

Aus dem Gesagten folgt auch, daß, solange das fragliche Gesetz seine Gültigkeit besitzt, die Zerstörung von Enzym, wenn eine solche überhaupt stattfindet, nach dem Verlauf von der gleichen Anzahl von $\mathrm{p} \cdot \mathrm{t}$ der zugegebenen Enzymmenge proportional sein muß.

Ob etwas von dem Enzym in dem im Reaktionsgemisch vorhandenen Wasser bleibt, ohne von irgend welcher anderen Substanz aufgenommen zu werden, ist auch für die Gültigkeit des p · t-Gesetzes ohne Belang, wenn nur das Verteilungsgesetz auch für diesen Teil des Enzyms gültig bleibt.

Nach meinen früheren Untersuchungen ist die Regel, daß der Umsatz nach dem Verlauf der gleichen Anzahl von $p \cdot t$ derselbe ist, für die Einwirkung von Trypsin auf verschiedene Substrate gültig, nämlich Casein (in $0,2 \% \mathrm{Na}_{2} \mathrm{CO}_{3}$ gelöst), mit etwas $\mathrm{Na}_{2} \mathrm{CO}_{3}$ erhitztes und dann dialysierles Serumalbumin, erhitztes und dialysiertes Eierklar, dialysiertes Wittes Pepton. Die Regel behält ihre Gültigkeit auch bei sehr fortgeschrittener Verdauung. Wenn aber die Enzymmenge in einer der zu vergleichenden Proben so groß ist, daß das Substrat mit Enzym völlig gesättigt wird und außerdem noch etwas Enzym frei bleibt, dann kann die Verteilung des Enzyms nicht von deren Menge unabhängig geschehen. In solchen Fällen muß eine Probe mit viel Enzym einen geringeren Umsatz zeigen als eine Probe mit weniger Enzym und entsprechend längere Zeit der Einwirkung. Dies stimmt auch völlig mit der Erfahrung überein. ${ }^{1}$ )

In der letzten Zeit habe ich einige neue Versuche über die Gültigkeit des oben behandelten Enzymzeitgesetzes angestellt. Es hat sich erwiesen, daß die Regel für gewisse Substratmischungen nicht länger gültig ist. Im folgenden Versuch

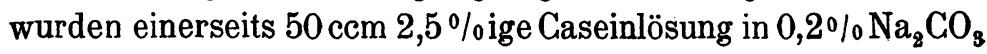
mit Trypsin verdaut, anderseits die gleiche Caseinmenge zu-

1) Journ. of Physiol., Bd. XXXII, S. 483, 1905. 
sammen mit $15 \mathrm{ccm}$ dialysierter Eiklarlösung. Nach beendeter Verdauung wurde der Umsatz durch N-Bestimmung in gleichen Volumina des Gerbsäurefiltrates ermittelt; die Anzahl Kubikzentimeter 1/10-n-Säure, die zur Neutralisation des Ammoniaks erforderlich waren, sind in die Tabelle eingetragen. Unter A sind die mit Caseinlösungen: $50 \mathrm{ccm}$ Casein $+45 \mathrm{ccm}$ (Trypsin $+\mathrm{H}_{2} \mathrm{O}$ ) und wechselnden Trypsinmengen erhaltenen Werte aufgenommen; unter B die mit $50 \mathrm{ccm}$ Casein + $15 \mathrm{ccm}$ Eierklar $+30 \mathrm{ccm}\left(\right.$ Trypsin $+\mathrm{H}_{2} \mathrm{O}$ ) erhaltenen:

\begin{tabular}{c|c|c|c}
\hline $\begin{array}{c}\text { Enzymmenge } \\
\mathrm{ccm}\end{array}$ & $\begin{array}{c}\text { Zeit } \\
\text { Stunden }\end{array}$ & A. & B. \\
\hline 30 & 3 & 27,5 & 21,3 \\
15 & 6 & 27,6 & 17,25 \\
10 & 9 & 27,5 & 13,18
\end{tabular}

Für diejenigen Fälle, wo das Casein allein als Substrat diente, war also das Enzym-Zeitgesetz gültig, was nach der obigen Betrachtungsweise bedeutet, daß das Trypsin auf das Casein und dessen Spaltungsprodukte unabhängig von der Menge des Trypsins in gleicher Weise sich verteilt. Für die Proben, welche außer Casein noch Eierklar enthielten, war das EnzymZeitgesetz nicht gültig. Das zugesetzte Eierklar würde allein mit den gebrauchten Trypsinmengen in den entsprechenden Zeiten keine nachweisbaren Veränderungen erlitten haben. Trotzdem wirkt es auf die Verdauung des Caseins hemmend. Dies geht einerseits aus den obigen Bestimmungen hervor, anderseits habe ich eine solche Hemmung in einer früheren Arbeit nachgewiesen. ${ }^{1}$ ) Das Eierklar nimmt also einen Teil des Trypsins an sich. Nun ist es ohne weiteres klar, daß, wenn das Enzym unabhängig von deren Menge zwischen dem Casein und dem Eierklar in gleicher Weise verteilt würde, das oben behandelte Enzym-Zeitgesetz auch für diejenigen Proben ihre Gültigkeit behalten würde, welche zur selben Zeit Casein und Eierklar erhalten. Dies war nicht der Fall. Der Versuch zeigt, daß die Verdauung des Caseins eine kräftigere Hemmung erfuhr, je

1) Diese Zeitschrift, Bd. LII, S. 412, 1907. 
weniger Trypsin zugegen war. Dies bedeutet, daß das Eierklar verhältnismäßig mehr Trypsin an sich nimmt, je geringer die zugesetzte Trypsinmenge war. Die Verteilung geschieht also nicht wie zwischen zwei Lösungsmitteln, sondern mehr wie bei der Adsorption. Bei der Adsorption wird nämlich von einer geringen Menge adsorbierbarer Substanz verhältnismäßig mehr adsorbiert als von einer größeren. In diesem Falle wäre also das Eierklar der adsorbierende Stoff ; das Casein wäre mit dem Lösungsmittel der zu adsorbierenden Substanz (des Trypsins) zu vergleichen. In Ermangelung genauer Messungen über die Verteilung des Trypsins läßt sich aber keine Formel für die Verteilung aufstellen. Wie ich schon vorher hervorgehoben habe, nimmt das Eierklar das Trypsin in reversibler Weise auf und nicht wie das native Serumalbumin, das den größten Teil des aufgenommenen Trypins an sich verfestigt oder vielleicht zerlegt. ${ }^{1}$ )

\section{Zusammenfassung.}

Bei der Verdauung des Caseins mit Trypsin hat es sich herausgestellt, daß, wenn bei gleicher Substratmenge die Zeit der Einwirkung der zugesetzten Enzymmenge umgekehrt proportional variiert wird, der gleiche Umsatz erhalten wird. Dies bedeutet, daß der Geschwindigkeitskoeffizient der Reaktion der Enzymmenge proportional ist, oder daß das Verhältnis zwischen den von den verschiedenen Stoffen aufgenommenen Trypsinmengen konstant bleibt unabhängig von der Menge des zugesetzten Trypsins.

Wenn aber Casein zusammen mit Eierklar der Trypsinverdauung unterworfen wird, geschieht die Verteilung in anderer Weise. Das Eierklar nimmt nämlich von einer geringen Trypsinmenge verhältnismäßig mehr an sich als von einer größeren, etwa so wie es bei der Adsorption der Fall ist.

Upsala, den 11. September 1908.

1) Diese Zeitschrift, Bd. LII, S. 412, 1907. 